\title{
A Combined Approach Effectively Enhancing Traffic Performance for HSR Protocol in Smart Grids
}

\author{
Nguyen Xuan Tien ${ }^{1}$, Jong Myung Rhee ${ }^{1}$ and Sang Yoon Park ${ }^{2, *}$ \\ 1 Department of Information and Communications Engineering, Myongji University, 116 Myongji-ro, Yongin, \\ Gyeonggi 17058, Korea; tiennguyen@mju.ac.kr (N.X.T.); jmr77@mju.ac.kr (J.M.R.) \\ 2 Department of Electronic Engineering, Myongji University, 116 Myongji-ro, Yongin, Gyeonggi 17058, Korea \\ * Correspondence: sypark@mju.ac.kr; Tel.: +82-31-330-6751
}

Received: 4 August 2017; Accepted: 5 September 2017; Published: 8 September 2017

\begin{abstract}
In this paper, we propose a very effectively filtering approach (EFA) to enhance network traffic performance for high-availability seamless redundancy (HSR) protocol in smart grids. The EFA combines a novel filtering technique for QuadBox rings (FQR) with two existing filtering techniques, including quick removing $(\mathrm{QR})$ and port locking (PL), to effectively reduce redundant unicast traffic within HSR networks. The EFA filters unicast traffic for both unused terminal rings by using the PL technique and unused QuadBox rings based on the newly-proposed FQR technique. In addition, by using the QR technique, the EFA prevents the unicast frames from being duplicated and circulated in rings; the EFA thus significantly reduces redundant unicast traffic in HSR networks compared with the standard HSR protocol and existing traffic filtering techniques. The EFA also reduces control overhead compared with the filtering HSR traffic (FHT) technique. In this study, the performance of EFA was analyzed, evaluated, and compared to that of the standard HSR protocol and existing techniques, and various simulations were conducted to validate the performance analysis. The analytical and simulation results showed that for the sample networks, the proposed EFA reduced network unicast traffic by $80 \%$ compared with the standard HSR protocol and by $26-62 \%$ compared with existing techniques. The proposed EFA also reduced control overhead by up to $90 \%$ compared with the FHT, thus decreasing control overhead, freeing up network bandwidth, and improving network traffic performance.
\end{abstract}

Keywords: smart grids; substation automation system (SAS); high-availability seamless redundancy (HSR); seamless communications; traffic reduction technique

\section{Introduction}

The smart grid is a new concept of the next-generation electric power system that has emerged to address challenges of the existing power grid. The smart grid is a modern electric power grid infrastructure for enhanced efficiency, reliability and safety, with smooth integration of renewable and alternative energy sources, through automated control and modern communications technologies [1]. Communication architecture of smart grid including requirements, topologies, and protocol stack is required for smart grid environments [2]. Smart grids provide more electricity to meet rising demand, increase reliability and quality of power supplies, increase energy efficiency, be able to integrate low carbon energy sources into power networks [3]. To realize the smart grid technology, legacy substations are retrofitted by substation automation systems (SASs), allowing the robust control and communication tasks [4]. The SAS system is used to control, supervise, and protect substations that are strategic nodes in power networks, which consist of large numbers of switchgears and measuring devices [5]. Functions of an SAS are control and supervision, as well as protection and monitoring of the primary equipment and of the grid. The functions can be assigned to three levels: the station level, the bay level, and the process level. Devices of an SAS may be physically 
installed on different functional levels [5]. The substation should continue to be operable if any SAS communications component fails [6]. Communication networks in the SAS are defined by the International Electrotechnical Commission (IEC)'s IEC 61850 standard [5,6]. To ensure the operations of substations, seamless communication with fault-tolerance can be provided for SASs by using redundancy protocols. In most redundancy protocols, the communication is recovered from a fault in the network; this recovery takes some time, and even a short time period could be unacceptable for certain time-critical applications, including SAS [7]. To help with this problem, the IEC developed and standardized two protocols that provide seamless communication with fault tolerant capability, including the parallel redundancy protocol (PRP) and high-availability seamless redundancy (HSR) [8]. Both these protocols implement redundancy in the nodes and provide seamless communication based on the duplication of frames sent over redundant paths from a source to a destination. Unlike the PRP that uses two identical local area network (LAN) of similar topology, the HSR is applied to a single LAN to provide seamless redundancy for the network. With respect to PRP, HSR allows to roughly halve the network infrastructure [8]. HSR is one of the redundancy protocols selected to provide seamless communication for SASs. HSR is mainly used ring topologies, including single-ring and connected-ring networks. In HSR, a node has two ports operated in parallel, called a doubly-attached node with HSR protocol (DANH). Singly-attached nodes (SANs), such as maintenance laptops or printers, cannot be inserted directly into HSR rings. SANs communicate with HSR ring devices through a redundancy box (RedBox) that acts as a proxy for the SANs attached to it. Quadruple port devices (QuadBoxes) are used to connect DANH rings in connected-ring networks. A single DANH ring consists of DANHs, each having two ring ports, interconnected by full-duplex links. When a source DANH sends a unicast frame to a destination DANH in a DANH ring, the source DANH prefixes the frame by an HSR tag and sends the tagged frame over each port. In the failure-free case, the destination DANH receives two identical frames from each port, removes the HSR tag of the first frame before passing it to its upper layers, and discards any duplicate. The standard HSR protocol works very effectively in single-ring networks. The HSR protocol, however, generates too much redundant unicast traffic in connected-ring networks. This drawback is caused by the following issues:

1. Issue 1: Duplicating and circulating frames in all the rings, except the destination DANH ring;

2. Issue 2: Forwarding unicast frames into all DANH rings;

3. Issue 3: Forwarding unicast frames into all QuadBox rings.

Several traffic filtering techniques have been proposed to solve the drawback and improve the network performance in HSR networks, including the quick removing $(\mathrm{QR})$ technique [9], the traffic control (TC) technique [10], the port locking (PL) technique [11], the hybrid QR and PL approach (QRPL) [12], the enhanced port locking (EPL) technique [13], and the filtering HSR traffic (FHT) technique [14]. However, most of the techniques (QR, TC, PL, QRPL, and EPL) do not solve all the HSR issues, whereas the FHT solves all the issues but generates additional control overhead in HSR networks. Several dual paths-based techniques have been proposed to reduce redundant unicast traffic in HSR networks based on pre-established paths. These techniques discover and establish dual paths between a source and a destination in an HSR network before forwarding unicast traffic frames from the source to the destination through the dual paths. Dual paths-based techniques include the dual virtual paths (DVP) [15] technique, which was then extended as extended dual virtual paths (EDVP) [16], the ring-based dual paths (RDP) [17] technique, and the dual separate paths (DSP) [18] technique. These dual paths-based techniques significantly reduce redundant unicast traffic in HSR networks. The main drawback of the techniques, however, is to generate additional control overhead in the networks because they exchange control messages to discover and establish dual paths. In addition, there are other techniques for reducing redundant unicast traffic in HSR networks, including the HSR SwitchBox technique [19] and the integration of HSR and OpenFlow (HSE + OF) [20]. The HSR SwitchBox technique defines a new switching node in HSR networks that forwards HSR frames based on looking up of media access control (MAC) tables instead of flooding 
the frames. The HSE + OF approach aims to manage HSR networks by means of the software defined networking (SDN) paradigm. The approach defines new HSE + OF nodes whose control plane is managed by an OpenFlow controller [20]. In other words, this approach is an implementation of HSR in SDN.

In this paper, we propose a very effectively filtering approach (EFA) to significantly reduce redundant unicast traffic in HSR networks by combining two existing filtering techniques with a newly-proposed filtering technique. We propose a new traffic filtering technique for QuadBox rings (FQR) to filter unicast traffic for unused QuadBox rings. The FQR technique is then combined with two existing techniques, including PL and QR to provide the combined approach EFA that effectively reduces redundant unicast traffic in HSR networks. The proposed EFA filters unicast traffic for both unused DANH rings by using the PL technique and unused QuadBox rings by using the newly-proposed FQR technique. The EFA also prevents the unicast traffic frames from being duplicated and circulated in rings by using the QR technique. The EFA, thus, significantly reduces redundant unicast traffic in HSR networks compared with the standard HSR protocol and other traffic-filtering techniques, such as QR, PL, QRPL, and EPL. In addition, although the EFA, FHT, and dual paths-based techniques exhibit similar network traffic performance, the EFA generates less control overhead than the FHT technique and existing dual paths-based techniques. In other words, the motivation of this paper is to propose a novel approach that reduces more redundant unicast traffic than the existing filtering techniques and generates less control overhead than the FHT and existing dual paths-based techniques. The proposed approach, therefore, saves network bandwidth and improves network performance.

The rest of this paper is organized as follows: Section 2 describes several existing traffic filtering techniques, while Section 3 introduces the proposed EFA for effectively reducing unicast traffic in HSR networks. In Section 4, the performance of the proposed approach is analyzed, evaluated, and compared to that of the standard HSR protocol and existing techniques. Section 5 describes several simulations and their results in order to evaluate and validate the performance analysis. Finally, Section 6 provides several conclusions drawn from this work.

\section{Related Work}

This section introduces several existing traffic filtering techniques including QR, PL, QRPL, EPL, and FHT that were proposed to reduce redundant unicast traffic in HSR networks. To demonstrate the operations of these existing filtering techniques, we consider an HSR network with eight DANH rings and three QuadBox rings as shown in Figure 1.

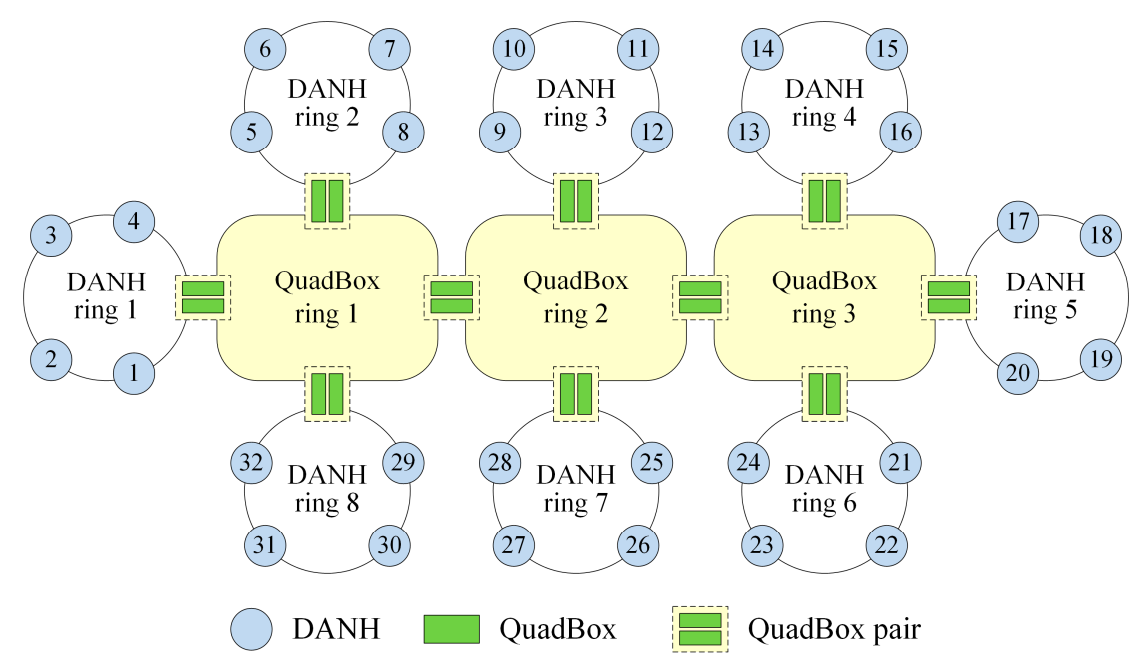

Figure 1. A high-availability seamless redundancy (HSR) network with eight doubly-attached node with HSR protocol (DANH) rings. 
Under the standard HSR protocol, when source node 1 sends a unicast frame to destination node 10, the frame is duplicated and circulated in all rings, except the destination DANH ring as shown in Figure 2. This flooding process generates excessively redundant unicast traffic in the HSR network.

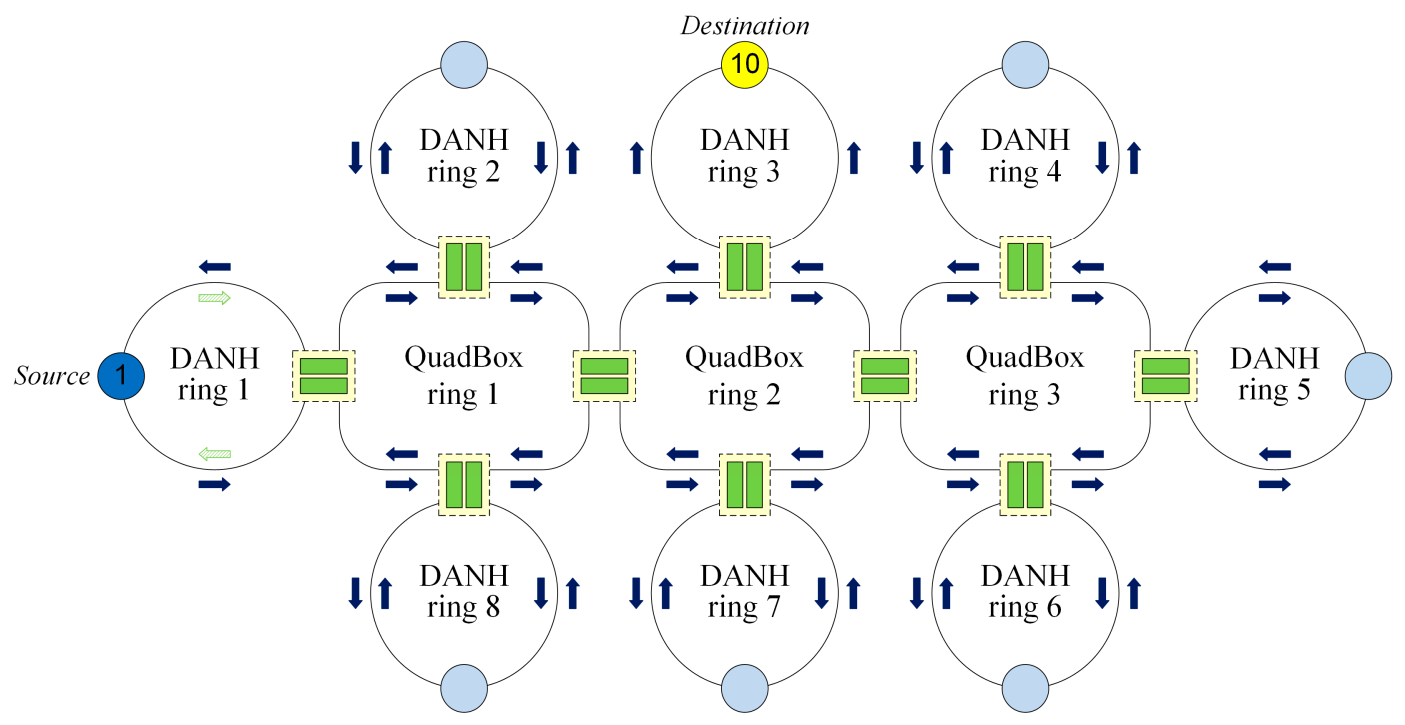

Figure 2. Forwarding a unicast frame using the standard HSR protocol.

\section{1. $Q R$}

QR reduces redundant traffic in HSR networks by solving the HSR issue 1. In other words, the QR technique prevents traffic frames from being duplicated and circulated in rings, as shown in Figure 3. When a node receives an HSR frame for the first time, the node floods the frame over all its ports except the received port from which the frame has been received. Later, if the node continues to receive other copies of the frame, the node will discard the duplicate copies.

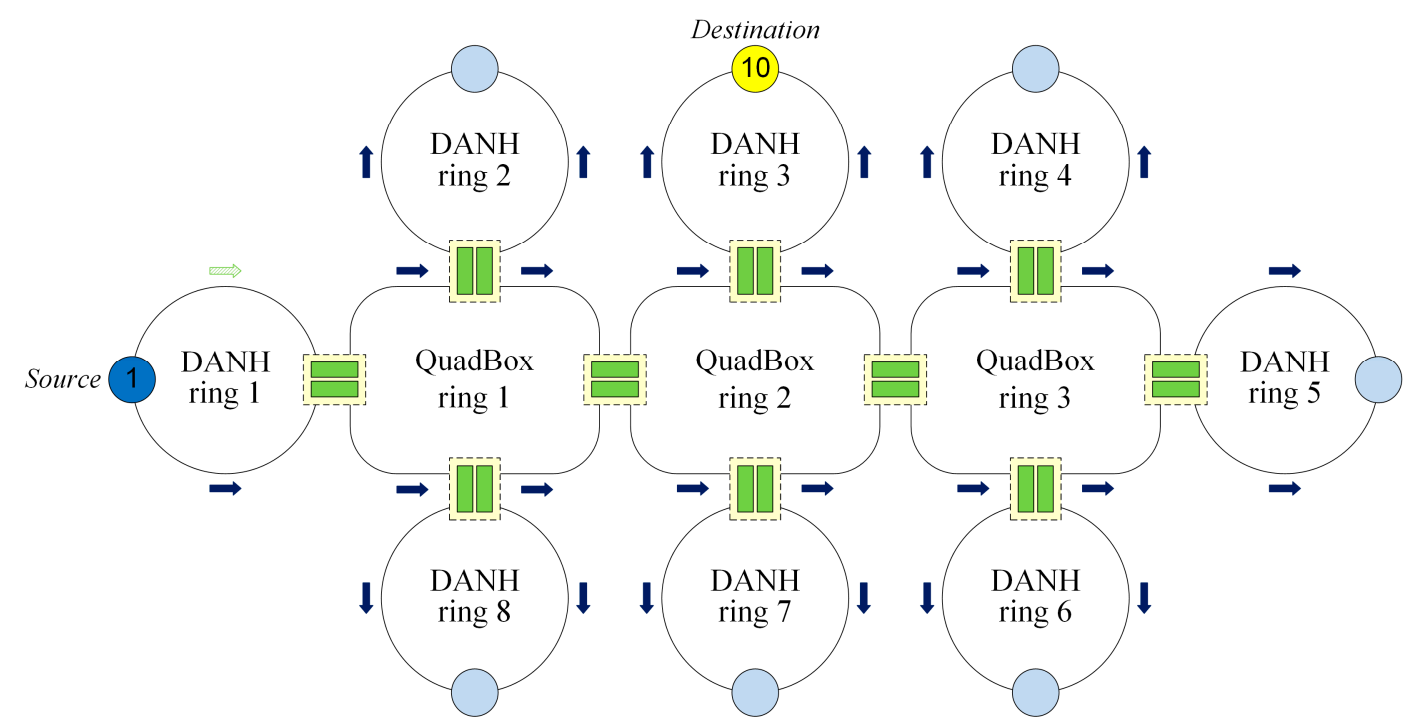

Figure 3. Forwarding a unicast frame using the quick removing $(\mathrm{QR})$ technique.

$\mathrm{QR}$ is the simplest traffic filtering technique. The technique can filter any traffic. The main disadvantage of QR, however, is that it does not filter unicast traffic for unused rings. 


\section{2. $P L$}

PL reduces redundant unicast traffic in HSR networks by filtering unicast traffic for unused DANH rings. PL divides each access QuadBox into two sides: a DANH side that is connected to a DANH ring and a QuadBox side that is connected to a QuadBox ring. The technique does not forward a unicast frame to DANH rings that do not contain the destination DANH by locking DANH sides connecting the DANH rings. When the source DANH sends unicast frames to the destination DANH, the PL technique uses the first sent frame to check if DANH rings contain the destination DANH and then lock DANH rings that do not contain the destination DANH. After the phase, the PL does not forward unicast frames into the locked DANH rings, as shown in Figure 4.

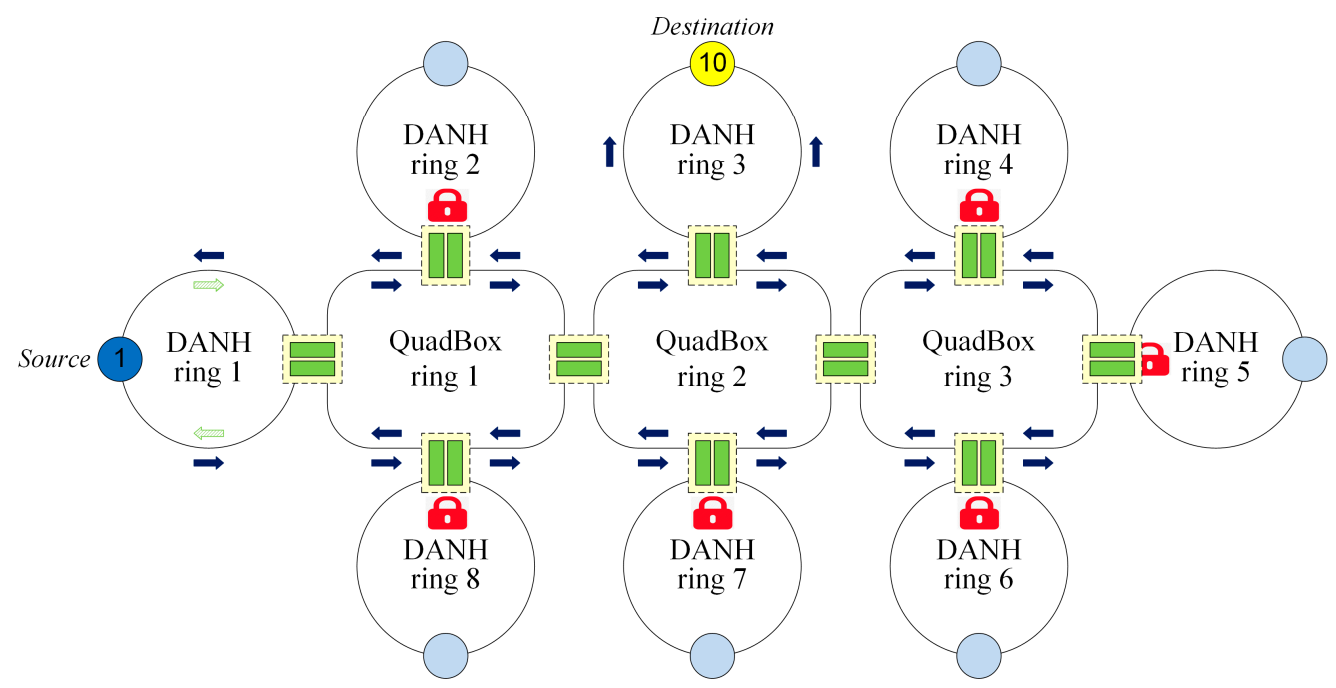

Figure 4. Forwarding a unicast frame using the port locking (PL) technique.

The drawbacks of PL, however, are that it does not solve the HSR issues 1 and 3.

\subsection{QRPL}

QRPL is a hybrid approach that combines the QR technique with the PL technique. The QRPL approach uses the PL technique to filter unicast traffic frames for DANH rings and the QR technique to remove duplicated and circulated frames from rings, as shown in Figure 5.

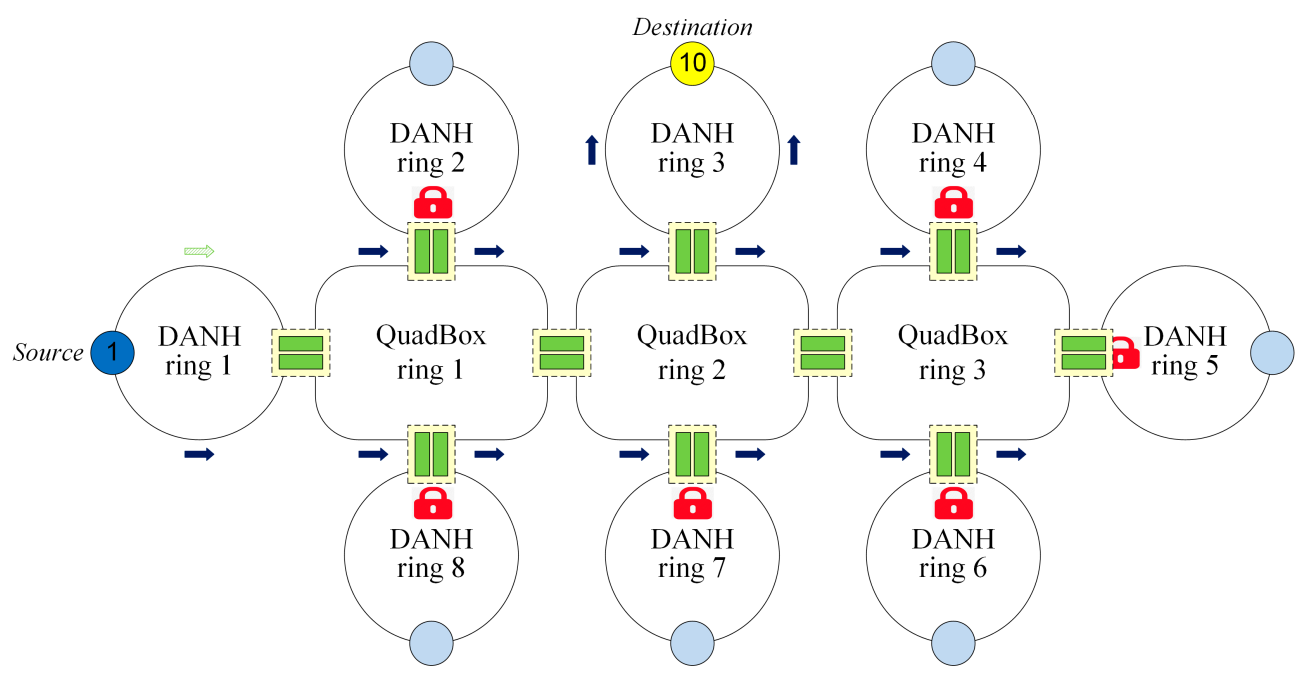

Figure 5. Forwarding a unicast frame using the hybrid QR and PL (QRPL) approach. 
The main drawback of the QRPL approach, however, is that it does not filter unicast traffic for QuadBox rings, which results in redundant unicast traffic traveling in these rings.

2.4. $E P L$

EPL is the improved version of the PL technique that filters unicast traffic for both unused DANH and QuadBox rings, as shown in Figure 6. The EPL technique works with the same locking concept as the PL technique.

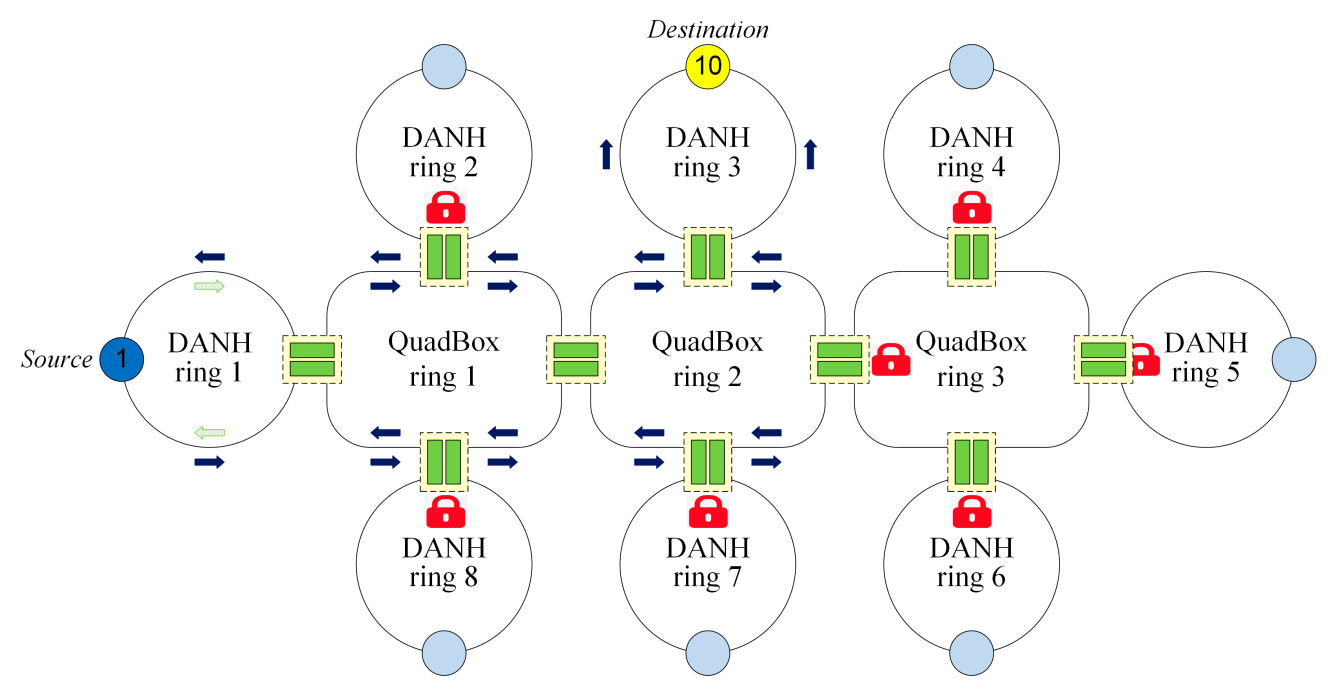

Figure 6. Forwarding a unicast frame using the enhanced port locking (EPL) technique.

The EPL technique, however, does not prevent unicast frames from being duplicated and circulated in active rings.

\subsection{FHT}

FHT is the only technique that solves all the HSR issues abovementioned in Section 1. The FHT technique filters unicast frames for both unused DANH and QuadBox rings by using MAC tables. The technique learns MAC addresses of DANHs and builds MAC tables by exchanging control messages. In addition, the FHT prevents unicast frames from being duplicated in rings. The process of forwarding unicast frames under the FHT technique is shown in Figure 7.

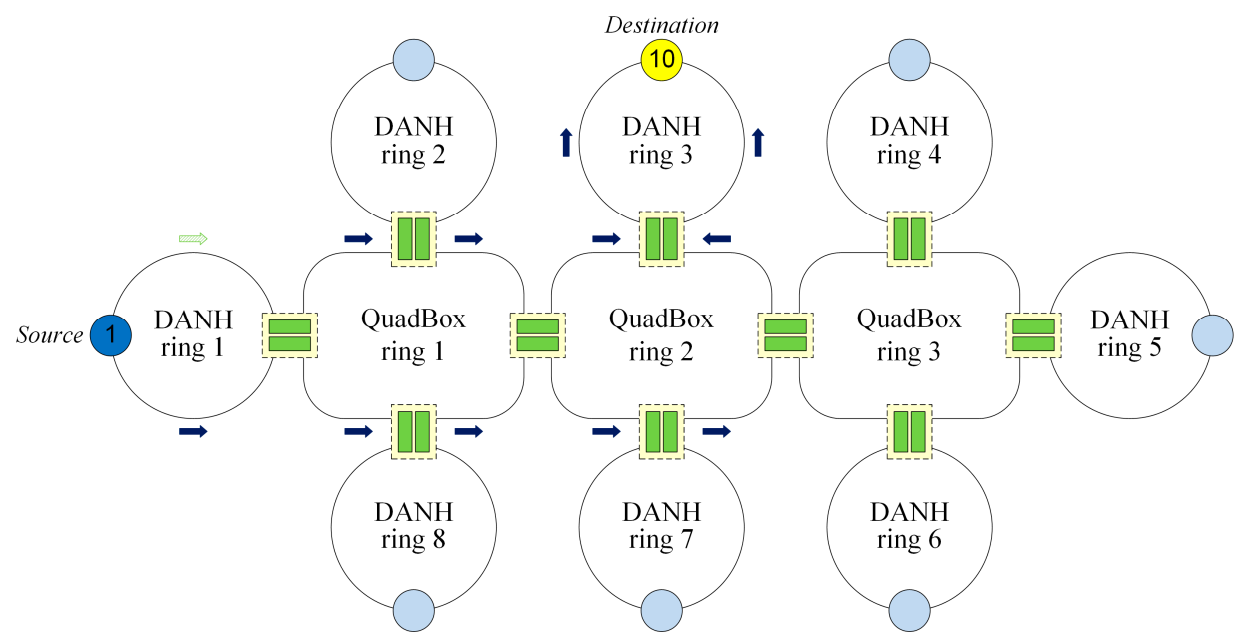

Figure 7. Forwarding a unicast frame using the filtering HSR traffic (FHT) technique. 
The main drawback of the FHT technique is that it generates additional control overhead in HSR networks due to the use of control messages.

\subsection{Comparisons}

As described above, several existing traffic filtering techniques have advantages and disadvantages. While QR prevents unicast frames from being duplicated and circulated in rings, it does not filter unicast traffic for unused rings. The PL and EPL filter unicast traffic for unused rings. They, however, do not remove duplicated unicast traffic from rings. The QRPL is a hybrid approach that combines the QR with the PL in order to filter unicast traffic for unused DANH rings and to prevent the traffic from being duplicated and circulated in rings. The QRPL, however, does not filter the unicast traffic for unused QuadBox rings. The FHT filters unicast traffic for both unused DANH and QuadBox rings, as well as removing duplicated traffic from rings. FHT does generate a certain degree of control overhead for learning and building MAC tables, however. The characteristics of these filtering techniques are summarized in Table 1.

Table 1. Characteristics of traffic filtering techniques.

\begin{tabular}{cccccc}
\hline Features & QR & PL & QRPL & EPL & FHT \\
\hline Filter traffic for DANH rings & - & $\sqrt{ }$ & $\sqrt{ }$ & $\sqrt{ }$ & $\sqrt{ }$ \\
Filter traffic for QuadBox rings & - & - & - & $\sqrt{ }$ & $\sqrt{ }$ \\
Remove duplicated traffic & $\sqrt{ }$ & - & $\sqrt{ }$ & - & $\sqrt{ }$ \\
Generate control overhead & No & No & No & No & Medium \\
\hline
\end{tabular}

\section{The Proposed EFA}

Three types of QuadBoxes are defined in this paper: access QuadBoxes, trunk QuadBoxes, and QuadBox pairs:

- The access QuadBox is a QuadBox that connects to at least one DANH ring;

- The trunk QuadBox is a QuadBox that does not connect to any DANH ring; in other words, trunk QuadBoxes are used to connect QuadBox rings;

- A QuadBox pair refers to two QuadBoxes that are used as a pair to connect two rings to prevent a single point of failure.

The main purpose of the proposed EFA is to filter unicast traffic frames for both unused DANH rings and unused QuadBox rings, as well as preventing the unicast traffic frames from being duplicated and circulated in rings of HSR networks.

\subsection{Removing Duplicated Unicast Traffic}

To solve issue 1 of HSR, the EFA uses the QR technique to prevent unicast traffic frames from being duplicated and circulated in all rings of HSR networks. By using the QR technique, each HSR node, such as a DANH node or a QuadBox node, forwards a unicast frame over all its ports (except the received port) when the node has received the frame for the first time. Later, if the node receives copies of the frame, the node will discard the duplicated copies.

\subsection{Filtering Unicast Traffic for DANH Rings}

The EFA filters unicast traffic for any DANH rings that do not contain the destination by applying the PL technique. For a communication session between source node 1 and destination node 10, when the source sends the first unicast frame, each access QuadBox forwards the frame into its DANH ring to check if its DANH ring contains the destination node. If not, then the access QuadBox locks its DANH side to prevent the frame from being forwarded into the DANH ring. 


\subsection{Filtering Unicast Traffic for QuadBox Rings}

To solve issue 3 of HSR (as noted above), we propose in this paper a novel filtering technique called "filtering for QuadBox rings" (FQR) to filter unicast traffic for unused QuadBox rings. FQR divides each trunk QuadBox into two sides, both of which consist of two ports connected to a QuadBox ring. For a communication session between a source and a destination in an HSR network, FQR sets each side of each trunk QuadBox to either the source side or the destination side. The source side is closer to the source than the destination side, as shown in Figure 8. The source and destination sides of each trunk QuadBox are only locally significant in each communication session.

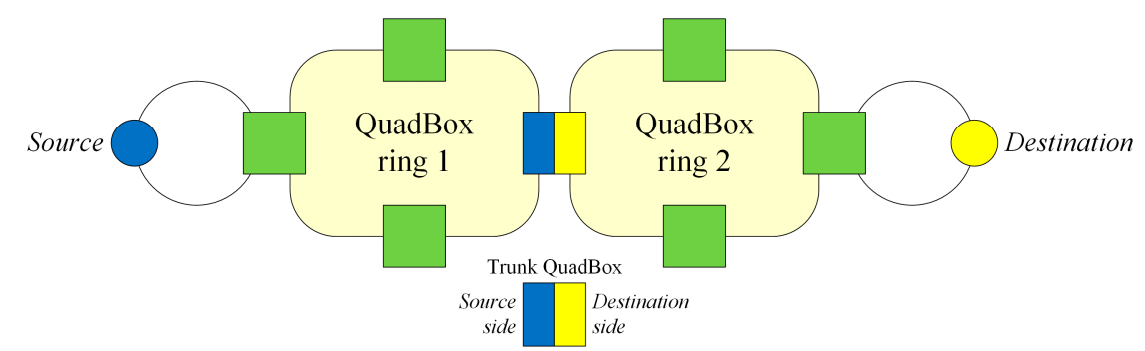

Figure 8. Source side and destination side of a trunk QuadBox.

FQR filters unicast traffic for any QuadBox rings that are not used to deliver unicast frames in a communication session between a source and a destination in an HSR network by locking the destination sides of the QuadBoxes. The FQR operation consists of (1) the setting step and (2) the locking step, as described below.

\subsubsection{Setting Step}

In this step, each side of each trunk QuadBox is set to either the source side or the destination side. This step is initiated once a communication session between a source and a destination is started. In other words, the step is performed while the first traffic frame is delivered from the source to the destination of the communication session. When the source sends the first HSR frame to the destination, the frame is flooded into all the rings in the network (as occurs in the standard HSR protocol). When a trunk QuadBox receives the first frame of the communication session, the trunk QuadBox sets the side from which the frame is received to the source side and sets the other side to the destination side, as shown in Figure 9.

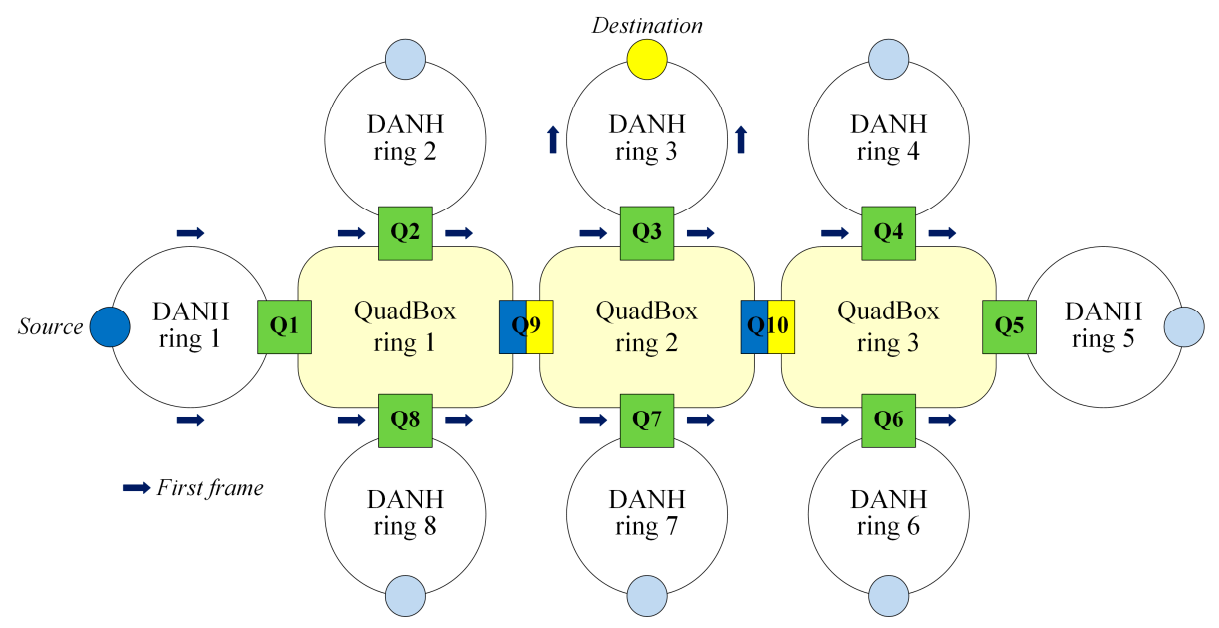

Figure 9. The process of the setting step. 


\subsubsection{Locking Step}

This step locks the destination sides of any QuadBoxes that are not used to deliver unicast traffic frames from the source to the destination. Once the destination receives the first HSR frame sent by the source, the destination initiates the locking step by sending a "locking" message back to the source. Only trunk QuadBoxes process and forward the locking message, whereas access QuadBoxes discard the message. When a trunk QuadBox receives a locking message, it checks if the side from that the locking message has been received is the source side or the destination side:

- If the received side is the destination side, then the QuadBox does not lock its destination side.

- If the received side is the source side, then the QuadBox locks its destination side.

The trunk QuadBox then forwards the received locking message over its ports, except for the received port and the locked ports, which belong to the locked destination side. Figure 10 shows the process of checking and locking destination sides based on receiving the locking message at trunk QuadBoxes.

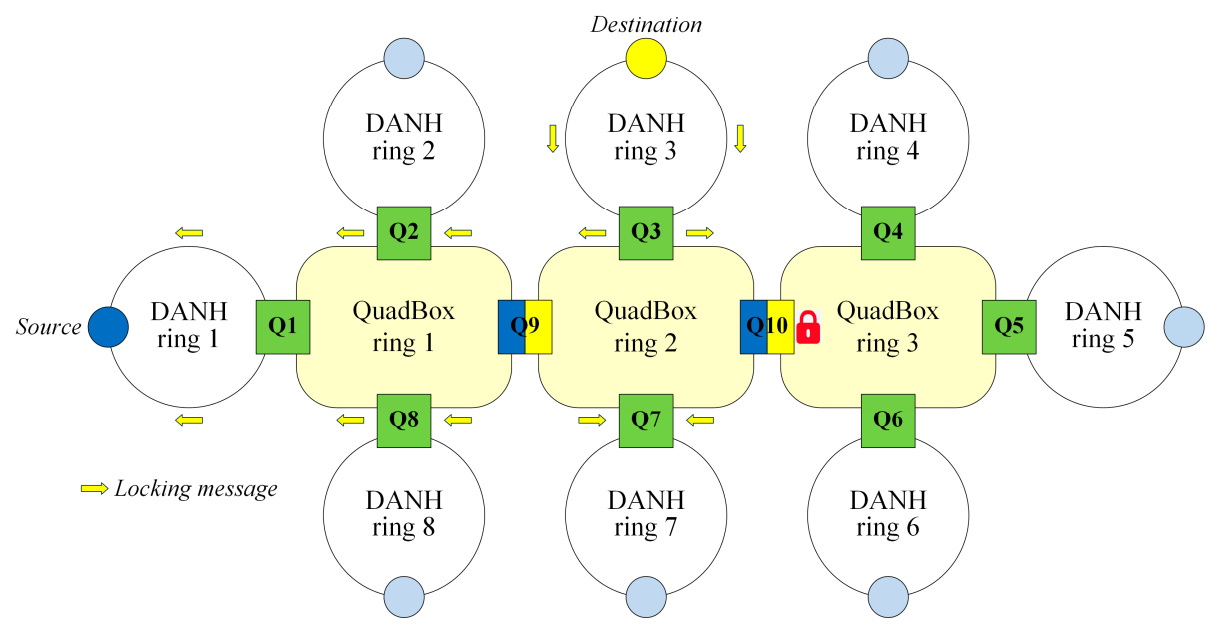

Figure 10. The process of the locking step.

\subsection{Operations of the Proposed EFA}

The EFA consists of two phases as shown in Figure 11, including locking phase and filtering phase. The locking phase occurs during the forwarding process of the first frame. In this phase, the EFA checks and locks access and trunk QuadBoxes that connect to unused DANH and QuadBox rings, respectively. The locking phase is followed by the filtering phase that starts when the second frame is sent. In the filtering phase, the EFA does not forward unicast frames into unused DANH and QuadBox rings.

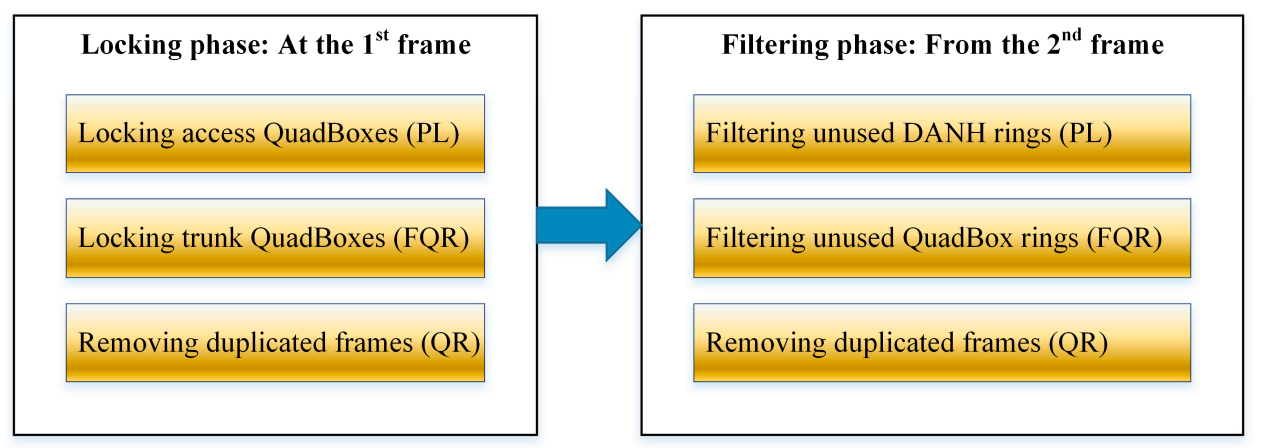

Figure 11. The operational diagram of effectively filtering approach (EFA). 
At the first frame, the network will work in the same way as the standard HSR protocol. From the second frame, unicast frames will be forwarded and filtered under the EFA effect. In the locking phase, when the source sends the first frame of a communication session to the destination, the frame is flooded in the whole network. During delivery of the first frame, access QuadBoxes use the PL technique to check their DANH rings and lock their DANH sides if their DANH rings do not contain the destination. Once the destination receives the first unicast frame, the destination sends a locking message back to the source. Upon receiving the locking message, trunk QuadBoxes use the FQR technique to check and lock their destination sides, if needed. Access QuadBoxes discard the locking message they have received. At that time, QuadBoxes have locked sides that are not used to forward unicast frames from the source to the destination. In the filtering phase, the next frames will not be forwarded into any DANH and QuadBox rings that are connected to locked DANH sides and locked destination sides. In addition, the EFA uses the QR technique to remove any duplicated unicast frames from rings. Figure 12 illustrates the process of forwarding unicast frames from source 1 to destination 10 in the sample HSR network.

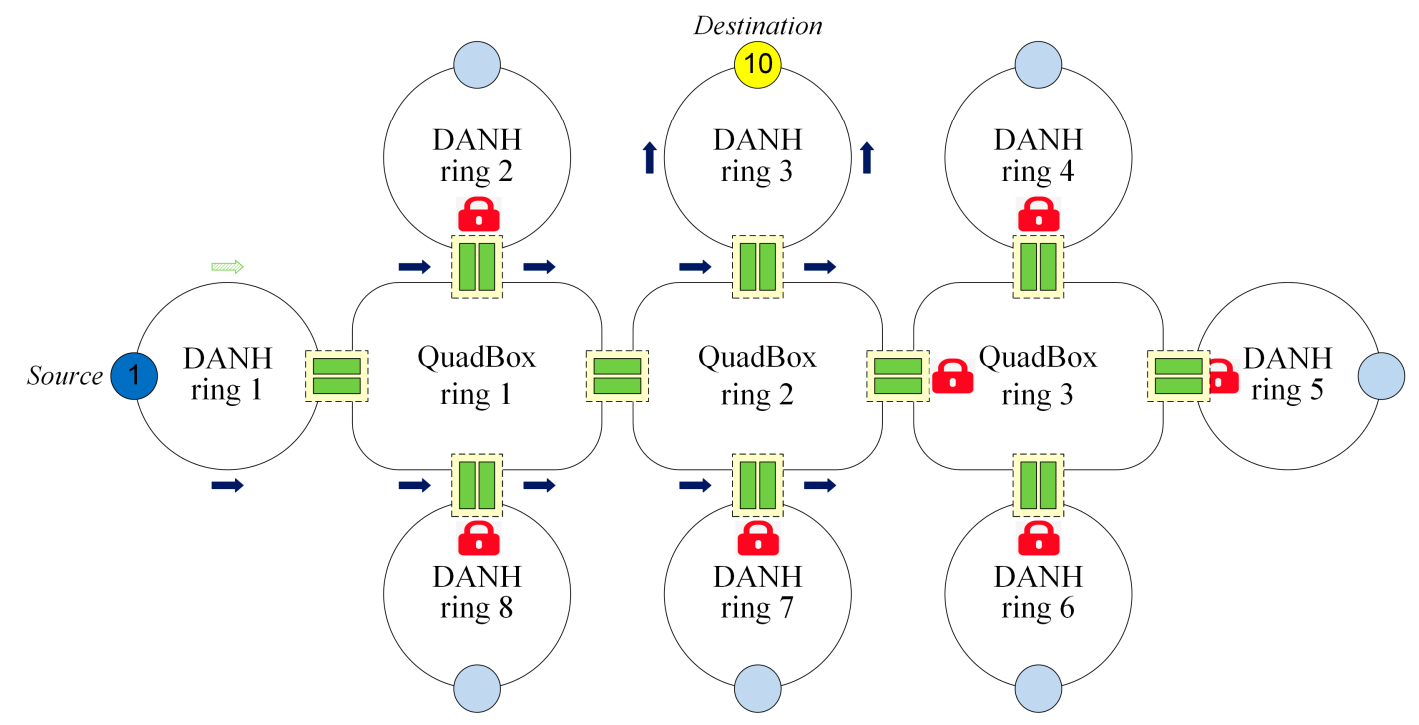

Figure 12. Forwarding a unicast frame using the EFA approach.

\section{Performance Analysis}

In this section, network traffic performance of EFA is first analyzed and compared with that of the standard HSR protocol and existing techniques. Control overhead performance of EFA is then analyzed and compared with that of the FHT technique.

\subsection{Network Traffic Performance}

This section describes the network traffic performance analysis of EFA compared to the standard HSR and existing techniques, including QR, PL, QRPL, and FHT.

In this paper, to analyze and evaluate traffic performance, network traffic was chosen as a performance metric. Network traffic is defined as the total number of frame copies that travel on links and that are received by nodes in the network. When a unicast frame is sent from a source to a destination in a network, the network traffic is the total number of the frame's copies that are delivered and received in the network. 


\subsubsection{Under the Standard HSR Protocol}

Let $n t_{H S R}^{1}$ be network traffic when a source node sends a unicast frame to a destination node under the standard HSR protocol. $n t_{H S R}^{1}$ is calculated as follows:

$$
n t_{H S R}^{1}=\sum_{i \in N R} 2 n_{i}-n_{D}
$$

where $n_{D}$ is the number of nodes in the destination DANH ring, $n_{i}$ is the number of nodes in the $i$ th ring, and $N R$ is a set of all rings in the network.

Generally, network traffic when a source node sends $N$ unicast frames to a destination node under the standard HSR protocol, denoted by $n t_{H S R}$, is determined by:

$$
n t_{H S R}=N\left(\sum_{i \in N R} 2 n_{i}-n_{D}\right)
$$

\subsubsection{Under the QR Technique}

When a source node sends a unicast frame to a destination node, network traffic under the QR technique, denoted by $n t_{Q R}^{1}$, is calculated by:

$$
n t_{Q R}^{1}=\sum_{i \in N R} n_{i}
$$

Generally, network traffic when a source node sends $N$ unicast frames to a destination node under the $\mathrm{QR}$ technique, denoted by $n t_{Q R}$, is determined as follows:

$$
n t_{Q R}=N \sum_{i \in N R} n_{i}
$$

\subsubsection{Under the PL Technique}

Network traffic when a source node sends the first unicast frame to a destination node under the PL technique, denoted by $n t_{P L^{\prime}}^{1}$, can be calculated using Equation (1):

$$
n t_{P L}^{1}=\sum_{i \in N R} 2 n_{i}-n_{D}
$$

Network traffic when the source node sends the $k$ th unicast frame $(k \geq 2)$ to the destination node under the PL technique, denoted by $n t_{P L}^{k}$, is determined as follows:

$$
n t_{P L}^{k}=\sum_{i \in Q R} 2 n_{i}+2 n_{S}+n_{D}
$$

where $n_{S}$ is the number of nodes in the source DANH ring and $Q R$ is a set of all QuadBox rings in the network.

Generally, network unicast traffic when a source node sends $N$ unicast frames to a destination node under the PL technique, denoted by $n t_{P L}$, is determined as follows:

$$
n t_{P L}=\sum_{i \in N R} 2 n_{i}+(N-1)\left(2 n_{S}+\sum_{i \in Q R} 2 n_{i}\right)+(N-2) n_{D}
$$




\subsubsection{Under the QRPL Approach}

Network traffic when a source node sends the first unicast frame to a destination node in an HSR network under the QRPL approach, denoted by $n t_{\mathrm{QRPL}}^{1}$, is similar to that under the QR technique and is calculated using Equation (3):

$$
n t_{Q R P L}^{1}=\sum_{i \in N R} n_{i}
$$

As with PL, starting from the second unicast frame sent in the HSR network, QRPL locks and filters all DANH rings except the destination DANH ring.

Network traffic when the source node sends the $k$ th unicast frame $(k \geq 2)$ to the destination node the QRPL, denoted by $n t_{\mathrm{QRPL}}^{k}$, is determined as follows:

$$
n t_{Q R P L}^{k}=\sum_{i \in Q R} n_{i}+n_{S}+n_{D}
$$

Generally, network unicast traffic when a source node sends $N$ unicast frames to a destination node under the QRPL, denoted by $n t_{Q R P L}$, is:

$$
n t_{Q R P L}=\sum_{i \in D R} n_{i}+N \sum_{i \in Q R} n_{i}+(N-1)\left(n_{S}+n_{D}\right)
$$

\subsubsection{Under the FHT Technique}

Network traffic when a source node sends a unicast frame to a destination node under the FHT technique, denoted by $n t_{F H T}^{1}$, is calculated as follows:

$$
n t_{F H T}^{1}=n_{S}^{D N}+n_{D}^{D N}+\sum_{i \in I R} n_{i}
$$

where $n_{S}^{D N}$ and $n_{D}^{D N}$ are the number of DANH nodes in the source and destination DANH rings, respectively, and $I R$ is a set of intermediate rings that connects the source and destination DANH rings.

Generally, network unicast traffic when a source node sends $N$ unicast frames to a destination node under the FHT technique, denoted by $n t_{F H T}$, is calculated as follows:

$$
n t_{F H T}=N\left(n_{S}^{D N}+n_{D}^{D N}+\sum_{i \in I R} n_{i}\right)
$$

\subsubsection{Under the EFA}

For the first unicast frame under the EFA, the process of delivering the frame is similar to that of the standard HSR protocol. During the delivering process, access and trunk QuadBoxes check and lock their DANH sides and destination sides, respectively, if the sides are not used to forward unicast frames from the source to the destination. Under the EFA, when a source node sends the first unicast frame to a destination node, the frame is flooded and doubled in all rings except the destination DANH ring. Network traffic, denoted by $n t_{E F A}^{1}$, is calculated as follows:

$$
n t_{E F A}^{1}=\sum_{i \in N R} 2 n_{i}-n_{D}
$$

where $n_{D}$ is the number of nodes in the destination DANH ring, $n_{i}$ is the number of nodes in the $i$ th ring, and NR is a set of all rings in the network.

For the next unicast frames, EFA locks all DANH rings except the destination DANH ring and any unused QuadBox rings. In addition, any duplicated frames are also removed from active rings. 
Network traffic when the source node sends the $k$ th unicast frame $(k \geq 2)$ to the destination node under the EFA, denoted by $n t_{E F A}^{k}$, is determined as follows:

$$
n t_{E F A}^{k}=n_{S}^{D N}+n_{D}^{D N}+\sum_{i \in A R} n_{i}
$$

where $n_{S}^{D N}$ and $n_{D}^{D N}$ are the number of DANH nodes in the source and destination DANH rings, respectively, and $A R$ is a set of active QuadBox rings that are used to deliver unicast frames between the source and the destination.

Generally, network traffic when a source node sends $N$ unicast frames to a destination node under the EFA, denoted by $n t_{E F A}$, is:

$$
n t_{E F A}=n t_{E F A}^{1}+(N-1) n t_{E F A}^{k}
$$

By substituting $n t_{E F A}^{1}$ in (13) and $n t_{E F A}^{k}$ in (14) into the above $n t_{E F A}$ equation, $n t_{E F A}$ can be calculated as:

$$
n t_{E F A}=\sum_{i \in N R} 2 n_{i}-n_{D}+(N-1)\left(n_{S}^{D N}+n_{D}^{D N}+\sum_{i \in I R} n_{i}\right)
$$

\subsection{Control Overhead Performance}

This section analyzes and evaluates the control overhead generated in HSR networks under the FHT and EFA.

\subsubsection{Under the FHT Technique}

The FHT filters unicast traffic for DANH and QuadBox rings based on MAC tables. To learn MAC addresses and build MAC tables, the FHT uses control messages, including Hello, ACK, and MAC messages.

Hello messages are sent by QuadBoxes and forwarded in DANH rings; DANH nodes forward the Hello messages, whereas QuadBoxes do not. The number of Hello messages sent and forwarded in the HSR network, denoted by $c o_{F H T}^{\mathrm{Hello}}$, is determined as follows:

$$
\operatorname{co}_{F H T}^{\text {Hello }}=\sum_{i \in D R}\left(n_{i}+1\right)+\sum_{i \in Q R} 2 n_{i}
$$

where $n_{i}$ is the number of nodes in the $i$ th ring, $D R$ is a set of all DANH rings, and $Q R$ is a set of all QuadBox rings in the network.

Upon receiving a Hello message, each DANH node sends an ACK message back to the QuadBox that sent the Hello message. The number of ACK messages sent and forwarded in the HSR network, denoted by $c o_{F H T}^{A C K}$, is determined as follows:

$$
c o_{F H T}^{A C K}=\sum_{i \in D R}\left(\frac{n_{i}^{D N}}{2}+1\right) \frac{n_{i}^{D N}}{2}
$$

where $n_{i}^{D N}$ is the number of DANH nodes in the $i$ th DANH ring.

Once an access QuadBox has built its MAC1 table, it sends a MAC message that contains all the MAC addresses of the access QuadBox's MAC1 table to its QuadBox ring. The trunk QuadBoxes connected to the QuadBox ring receive the MAC message, update their MAC2 tables, and then forward the MAC message. The number of MAC messages sent and forwarded in the HSR network, denoted by $c O_{F H T}^{M A C}$, is calculated as follows:

$$
c o_{F H T}^{M A C}=\sum_{i \in A Q} n_{i}^{Q R}
$$


where $A Q$ is a set of all access QuadBoxes in the network and $n_{i}^{Q R}$ is the number of QuadBoxes in the QuadBox ring connected to the $i$ th access QuadBox.

The total number of control messages sent and forwarded in the HSR network, denoted by $c o_{F H T}$, is determined as follows:

$$
c o_{F H T}=\sum_{i \in D R}\left(n_{i}+\sum_{i \in D R}\left(\frac{n_{i}^{D N}}{2}+1\right) \frac{n_{i}^{D N}}{2}\right)+\sum_{i \in Q R} 2 n_{i}+\sum_{i \in A Q} n_{i}^{Q R}
$$

\subsubsection{Under the EFA}

The EFA uses only one control message (the Locking message) to filter unicast traffic for QuadBox rings. Once the destination node receives the first frame sent by the source, the node sends a locking message back to the source. The locking message is then sent to the source through active rings (usually QuadBox rings) that are used to delivered unicast frames from the source to the destination. The locking message is not forwarded to the DANH rings.

Since the destination node sends the locking message once and the message is only forwarded in the destination DANH ring and active QuadBox rings, the total number of locking messages generated and forwarded in the HSR network, denoted by $c 0_{E F A}$, is calculated as follows:

$$
C o_{E F A}=n_{D}+\sum_{i \in A R} n_{i}
$$

where $n_{D}$ is the number of nodes in the destination DANH ring and $A R$ is a set of active QuadBox rings that connect the source and destination DANH rings.

It is clear that the EFA generates less control messages in HSR networks than the FHT.

\section{Simulations and Discussion}

To validate the analyzed performance and to evaluate the performance of the proposed EFA, various simulations were carried out using the OMNeT++ network simulator [21]. There are several common network simulation tools used to evaluate research results in communication networks, including Network Simulator 2 (ns-2) [22], Network Simulator 3 (ns-3) [23], Riverbed Modeler (formerly referred to as OPNET) [24], and OMNeT++. Each simulation tool has both benefits and drawbacks. Selecting a network simulator as a tool to evaluate results of a new research depends on the target of the research, the supporting ability of each network simulator, and the programming skills of each researcher. The primary application area of these network simulators is communication networks. All of these simulators can be used to analyze and evaluate the network performance of the traffic reduction techniques in HSR network. In this paper, OMNeT++ is selected as the network simulation tool to simulate and evaluate the proposed approach because the tool is one of the best network simulators and we are familiar with it.

In the simulations, the HSR network shown in Figure 1 was considered. The network consists of eight DANH rings and three QuadBox rings. Each DANH ring includes four DANHs.

\subsection{Simulation Description}

\subsubsection{Simulation 1}

The objective of the simulation was to validate and compare the network traffic performance of the EFA to that of the standard HSR protocol and existing traffic filtering techniques. In the simulation, source node 1 sends $N(N=10,20 \ldots 100)$ unicast frames to destination node 10. Network traffic frames were recorded to validate and compare with the analytical results. 


\subsubsection{Simulation 2}

The objective of the second simulation was to validate and compare the overhead performance of the EFA to that of the FHT technique. In this simulation, the sample HSR network shown in Figure 1 was used. Source node 1 sends $N(N=10,20 \ldots 100)$ unicast frames to destination node 10 . The total number of control messages generated in the network was recorded to validate and compare with the analytical results.

\subsection{Simulation Results}

\subsubsection{Simulation 1}

Figure 13 shows a comparison of the network traffic performance for the EFA, the standard HSR protocol, and several existing techniques, including QR, PL, QRPL, and FHT.

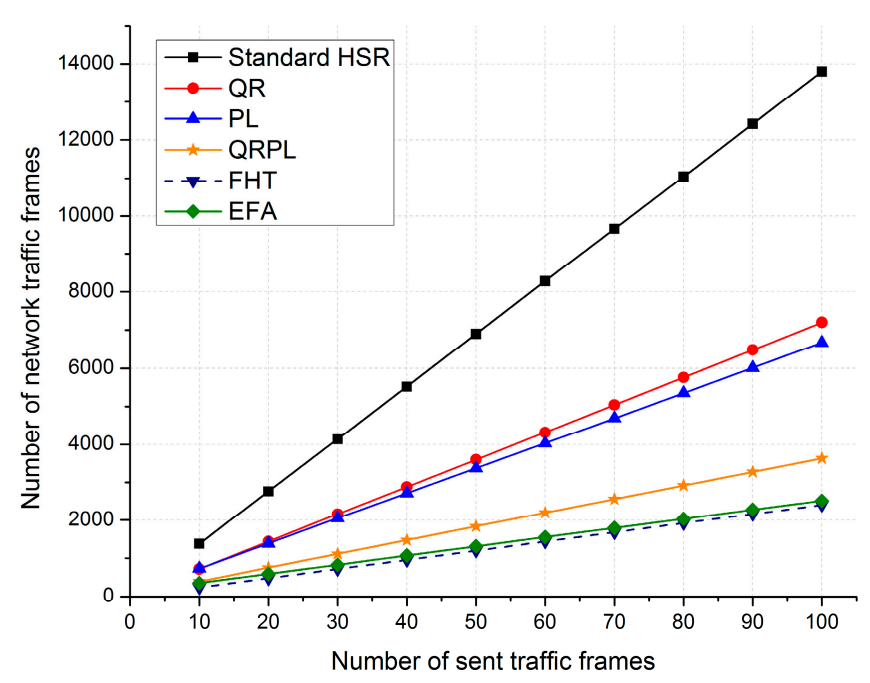

Figure 13. A comparison of network traffic performance.

The line graph in Figure 13 illustrates the number of network traffic frames recorded under traffic reduction techniques. The horizontal axis of the graph shows the number of unicast frames sent from the source to the destination, and the vertical axis shows the number of network traffic frames generated and delivered in the network when the source node sends the unicast frames to the destination node.

\subsubsection{Simulation 2}

Figure 14a shows a comparison of the control overhead performance between the EFA and the FHT technique; Figure 14b illustrates the control overhead messages to network traffic frames ratio of the EFA and FHT.

The line graph in Figure 14a illustrates the number of control messages recorded under the FHT and the EFA. The horizontal axis of the graph shows the number of unicast frames sent from the source to the destination, whereas the vertical axis shows the number of control messages generated and delivered in the network. The line chart in Figure 14b shows the control messages to network traffic frames ratio under the FHT and the EFA. 


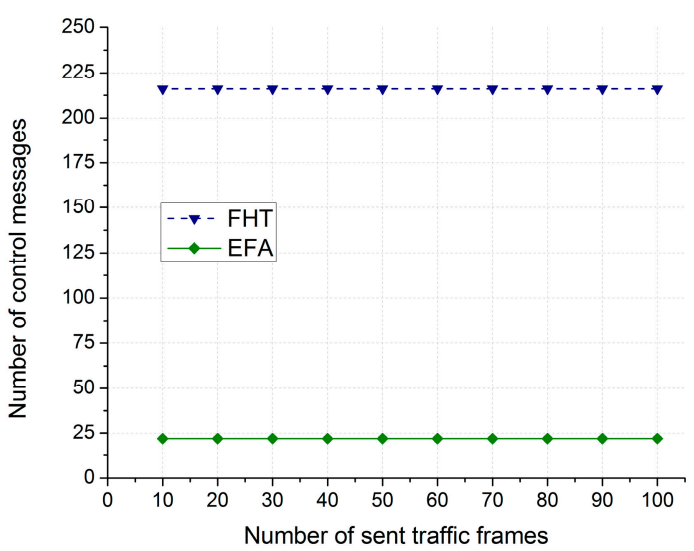

(a)

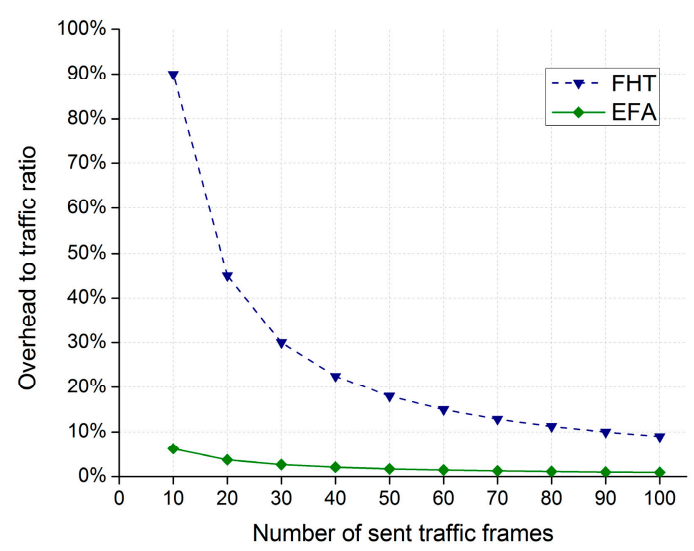

(b)

Figure 14. A comparison of overhead performance between the EFA and FHT. (a) Number of control messages; and (b) control messages to traffic frames ratio.

\subsection{Discussion}

The line graph in Figure 13 illustrates a comparison of network traffic of the EFA, the standard HSR protocol, and existing traffic-reduction techniques. Unlike other traffic-filtering techniques such as QR, PL, QRPL, and EPL—which either remove duplicate frames, and/or filter unicast traffic for DANH rings, or filter unicast traffic for both DANH and QuadBox rings-the EFA filters the unicast traffic for both DANH and QuadBox rings, as well as removing the duplicate frames from rings, as in the FHT technique. The EFA, thus, significantly reduces redundant unicast traffic compared with the standard HSR protocol and the existing QR, PL, and QRPL techniques. Numerically, for the sample HSR network, EFA reduces unicast network traffic by $80 \%$ compared with the standard HSR protocol, by $\sim 62 \%$ compared with the QR technique, by $\sim 60 \%$ compared with the PL technique, and by $\sim 26 \%$ compared with the QRPL approach.

The simulation results also demonstrate that the network traffic performances of the proposed EFA and the FHT are quite similar. The main drawback of EFA is that it still generates a few control messages that are used to check and lock trunk QuadBoxes connecting to unused QuadBox rings, resulting in additional control overhead in HSR networks. The EFA, however, has better control overhead performance than the FHT, as shown in Figure 14a,b. The line chart in Figure 14a shows that for the sample HSR network, the number of control messages generated by the FHT is ten times higher than that generated by the EFA.

Table 2 shows a comparison of filtering features of various traffic filtering-based techniques. It is clear from the table that the proposed EFA is one of the most effective solutions for reducing redundant unicast traffic in HSR networks.

Table 2. A comparison of features of traffic filtering-based techniques.

\begin{tabular}{ccccccc}
\hline Features & QR & PL & QRPL & EPL & FHT & EFA \\
\hline Filter traffic for DANH rings & - & $\sqrt{ }$ & $\sqrt{ }$ & $\sqrt{ }$ & $\sqrt{ }$ & $\sqrt{ }$ \\
Filter traffic for QuadBox rings & - & - & - & $\sqrt{ }$ & $\sqrt{ }$ & $\sqrt{ }$ \\
Remove duplicated traffic & $\sqrt{ }$ & - & $\sqrt{ }$ & - & $\sqrt{ }$ & $\sqrt{ }$ \\
Generate control overhead & No & No & No & No & Medium & Low \\
\hline
\end{tabular}

\section{Conclusions}

In this paper we proposed a novel approach called EFA for effectively reducing redundant unicast traffic in HSR networks. The EFA integrates a novel filtering technique for QuadBox rings in HSR networks called the FQR technique with two existing filtering techniques, $\mathrm{QR}$ and $\mathrm{PL}$, in order to filter 
redundant unicast traffic for both DANH and QuadBox rings, as well as to prevent unicast frames from being duplicated and circulated in rings. The EFA thus demonstrates the best network traffic performance among the various available filtering techniques. The analytical and simulation results showed that, for the sample network, EFA reduced network unicast traffic by $80 \%$ compared with the standard HSR protocol, by $\sim 62 \%$ compared with the QR technique, by $\sim 60 \%$ compared with the PL technique, and by $\sim 26 \%$ compared with the hybrid approach of QRPL. In addition, among the best traffic performance techniques, the EFA demonstrates the best overhead performance. For the sample network, the number of control messages generated by the EFA is one tenth that of the FHT. In conclusion, the EFA is a very efficient approach that reduces more redundant unicast traffic in HSR networks than existing filtering techniques and generates less control overhead in the networks than the FHT. The EFA, thus, saves network bandwidth and improves network traffic performance in HSR networks.

Our future work will develop and implement the EFA in hardware devices.

Acknowledgments: This work was partly supported by Institute for Information \& communications Technology Promotion (IITP) grant funded by the Korea government (MSIT) (No. 2014-0-00501, A Study on the Key Technology of Optical Modulation and Signal Processing for Implementation of $400 \mathrm{~Gb} / \mathrm{s}$ Optical Transmission) and Basic Science Research Program through the National Research Foundation of Korea (NRF) funded by the Ministry of Science and ICT (grant number: 2017R1A2B4003964).

Author Contributions: Authors Nguyen Xuan Tien, Jong Myung Rhee, and Sang Yoon Park conceived and developed the ideas behind the research. Nguyen Xuan Tien carried out the performance analysis and simulations, and wrote the paper under supervision of Jong Myung Rhee. Jong Myung Rhee, and Sang Yoon Park finalized the paper.

Conflicts of Interest: The authors declare no conflict of interest.

\section{References}

1. Güngör, V.C.; Sahin, D.; Kocak, T.; Ergüt, S.; Buccella, C.; Cecati, C.; Hancke, G.P. Smart grid technologies: Communication technologies and standards. IEEE Trans. Ind. Inform. 2011, 7, 529-539. [CrossRef]

2. Sauter, T.; Lobashov, M. End-to-end communication architecture for smart grids. IEEE Trans. Ind. Electron. 2011, 58, 1218-1228. [CrossRef]

3. ABB. What Is a Smart Grid? ABB Smart Grids. Available online: http://new.abb.com/smartgrids/what-isa-smart-grid (accessed on 2 August 2017).

4. Zeynal, H.; Eidiani, M.; Yazdanpanah, D. Intelligent Substation Automation Systems for Robust Operation of Smart Grids. In Proceedings of the 2014 IEEE Innovative Smart Grid Technologies-Asia (ISGT Asia), Kuala Lumpur, Malaysia, 20-23 May 2014.

5. IEC 61850-1 Standard: "Communication Networks and Systems in Substations_Part 1: Introduction and Overview"; IEC/TR 61850-1; The International Electrotechnical Commission: Geneva, Switzerland, 2003.

6. IEC 61850-3 Standard: "Communication Networks and Systems for Power Utility Automation-Part 3: General Requirements"; IEC 61850-3 Ed.2; The International Electrotechnical Commission: Geneva, Switzerland, 2010.

7. Araujo, J.A.; Lázaro, J.; Astarloa, A.; Zuloaga, A.; García, A. High Availability Automation Networks: PRP and HSR Ring Implementations. In Proceedings of the 2012 IEEE International Symposium on Industrial Electronics (ISIE), Hangzhou, China, 28-31 May 2012.

8. IEC 62439-3 Standard: "Industrial Communications Networks-High Availability Automation Networks-Part 3: Parallel Redundancy Protocol (PRP) and High-Availability Seamless Redundancy (HSR)"; IEC 62439-3 Ed.2; The International Electrotechnical Commission: Geneva, Switzerland, 2012.

9. Nsaif, S.A.; Rhee, J.M. Improvement of high-availability seamless redundancy HSR traffic performance for smart grid communications. J. Commun. Netw. 2012, 14, 653-661. [CrossRef]

10. Shin, M.; Joe, I. Performance Improvement for the HSR Ring Protocol with Traffic Control in Smart Grid. In Proceedings of the 2012 Future Generation Information Technology Conference (FGIT), Gangwon, Korea, 16-19 December 2012.

11. Abdulsalam, I.R.; Rhee, J.M. Improvement of High-Availability Seamless Redundancy (HSR) Unicast Traffic Performance Using Port Locking. In Proceedings of the Fourth World Congress on Software Engineering, Hong Kong, China, 3-4 December 2013. 
12. Altaha, I.R.; Rhee, J.M. Improvement to high-availability seamless redundancy (HSR) unicast traffic performance using a hybrid approach, QRPL. J. Satell. Inf. Commun. 2016, 11, 29-35.

13. Altaha, I.R.; Rhee, J.M.; Pham, H.A. Improvement of high-availability seamless redundancy (HSR) unicast traffic performance using enhanced port locking (EPL) approach. IEICE Trans. Commun. 2015, E98-D, 1646-1656. [CrossRef]

14. Tien, N.X.; Rhee, J.M. FHT: A novel approach for filtering high-availability seamless redundancy (HSR) traffic. Energies 2015, 8, 6249-6274. [CrossRef]

15. Nsaif, S.A.; Rhee, J.M. DVP: A novel high-availability seamless redundancy (HSR) protocol traffic-reduction algorithm for a substation automation system network. Energies 2014, 7, 1792-1810. [CrossRef]

16. Hong, S.; Lim, D.; Joe, I. Extended dual virtual paths algorithm considering the timing requirements of IEC61850 substation message type. IEICE Trans. Inf. Syst. 2016, E99.D, 1563-1575. [CrossRef]

17. Tien, N.X.; Kim, S.; Rhee, J.M. A novel ring-based dual paths approach for reducing redundant traffic in HSR networks. Comput. Netw. 2016, 110, 338-350. [CrossRef]

18. Tien, N.X.; Kim, S.; Rhee, J.M.; Park, S.Y. A novel dual separate paths (DSP) algorithm providing fault-tolerant communication for wireless sensor networks. Sensors 2017, 17, 1699. [CrossRef] [PubMed]

19. Tien, N.X.; Rhee, J.M. Developing a new HSR switching node (SwitchBox) for improving traffic performance in HSR networks. Energies 2016, 9, 36. [CrossRef]

20. Molina, E.; Jacob, E.; Toledo, N.; Astarloa, A. Performance enhancement of high-availability seamless redundancy (HSR) networks using OpenFlow. IEEE Commun. Lett. 2015, 20, 364-367. [CrossRef]

21. OMNeT++ v4.6 Discrete Event Simulator. Available online: http://www.omnetpp.org/ (accessed on 30 May 2017).

22. Network Simulator ns-2. Available online: https://www.isi.edu/nsnam/ns/ (accessed on 18 August 2017).

23. Network Simulator ns-3. Available online: https://www.nsnam.org/ (accessed on 18 August 2017).

24. River Modeler. Available online: https://www.riverbed.com/products/steelcentral/steelcentral-riverbedmodeler.html (accessed on 18 August 2017).

(C) 2017 by the authors. Licensee MDPI, Basel, Switzerland. This article is an open access article distributed under the terms and conditions of the Creative Commons Attribution (CC BY) license (http:/ / creativecommons.org/licenses/by/4.0/). 